\title{
Circular Polarization Antenna-Filter
}

\author{
${ }^{1}$ Kotelnikov Institute of Radio Engineering and Electronics, Russia \\ ${ }^{2}$ Moscow Design Bureau "Kompas," Russia \\ ${ }^{3}$ Moscow Power Engineering Institute, Russia \\ E-mail: sbankov@yandex.ru
}

Sergey Bankov', Alexander Davydov², Victor Kalinichev ${ }^{1}$, Peter Viugin ${ }^{2}$, Alexander Kurushin ${ }^{3}$

\begin{abstract}
This study investigated a two-function filtering antenna device in the 1-2 GHz band. The device radiates or receives electromagnetic waves of circular polarization and simultaneously performs the function of a second-order bandpass filter. The considered design consists of a dualmode printed antenna-resonator coupled with two nonradiating resonators, which are fed by a quadraturebalanced power divider. Using the model in the form of an equivalent circuit, the main features of the structure's synthesis and its tuning were analyzed, including potentially achievable characteristics. The results of the numerical simulations using electromagnetic software are presented in comparison with the experimental characteristics of the prototype, which was made of high-permittivity, highquality ceramics. This study found that the proposed design was characterized by an almost two-fold increase of the operating frequency band compared with the resonant antenna itself, while a low level of cross-polarization within the passband was simultaneously maintained. It also found that the design has the frequency response of a second-order bandpass filter.
\end{abstract}

\section{Introduction}

Recent years have seen increased interest in the research and development of multifunctional antennas. In particular, these include reconfigurable antennas and antenna-filters (AFs). The use of such structures makes it possible to expand the functionality of communication and navigation systems, reduce the size of transceiver modules, and improve the production technology.

In the traditional approach to a radio-frequency module design, an antenna and a bandpass filter are considered two different uncoupled devices performing different functions. The antenna provides coupling of the RF unit with free space through radiating or receiving electromagnetic waves, and it ideally does not contribute to the amplitude-frequency characteristic (AFC) of the unit. The filter shapes the required frequency response and does not radiate. However, the ever-increasing demands on the size of all RF elements are in conflict with this traditional concept. Indeed, reducing the antenna's size would inevitably lead to an increase of its Q-factor and, consequently, to a decrease of the operating frequency band [1]. As a result, the band requirements would not be satisfied for given volume limitations of the antenna, at least without the use of dissipative elements that reduce the antenna gain. A solution is to use reactive matching circuits that expand the bandwidth of the antenna. Because a small-size antenna is a sufficiently effective resonator and a matching circuit is a combination of narrowband resonators [2], the entire structure of an antenna with a matching circuit is a bandpass filter, which provides signal transmission in a given frequency band. In this case, there is no need to use a filter as an additional element because the frequency filtering function is performed directly in the antenna. Thus, the concept of an AF meets the increasing demands for the miniaturization of RF modules.

In general, AFs can be classified into two types of structures. The first type is a combination of an antenna and a filter. Electrically, they are uncoupled elements connected through their terminals. In structures of the second type, the antenna and filter are integrated both constructively and electrically. In this case, the antenna is one of the resonators of the composite radiating bandpass filter. In the authors' opinion, structures of the second type are of greater interest for applications. However, most work devoted to AFs has been related to linear-polarized structures of the first type. In [3], a single-layer filter on U-shaped resonators and a printed radiator were combined into a multilayer PCB. A similar construction was considered in [4], in which a Tshaped antenna and a filter made on coupled microstrip lines were used. A more complicated dual-band filter was studied in [5]. Its output was connected to the input of a complex shape radiator operating in two separated frequency bands. A circular polarization antenna with frequency-selective characteristics was considered in [6]. It uses a band-stop notch filter that suppresses higher-order resonances. The AF studied in [7] incorporated a filter made of four printed resonators of complex shape. This design provided the elliptic-type AFC with zeros of the transmission coefficient in the stop band, leading to a high level of signal suppression outside the bandpass.

Structures of the second type have also been presented quite widely (e.g., [8-12]). A linear-polarized AF was considered in [8], which comprised two square open-loop resonators, a coupled line, and a microstrip antenna. The antenna performed as not only a radiator but also the last 
resonator of the bandpass filter. The patch of the microstrip antenna was stacked above the system circuit board, and in this case was suitable for embedding inside an $\mathrm{RF}$ front end. In [9], a third-order AF with linear polarization was presented. An L-shaped antenna combined functions of a radiator and resonator of the filter. It represented a radiating resonator (RR), which was connected to two additional split-ring strip resonators. These strip resonators were made on a thin substrate and had insignificant radiation losses. They represented nonradiating resonators (NRR). Such an integration of RR and NRR is typical for AFs of the second type, and it can provide a stable radiation pattern in the operating frequency band.

Antennas of circular polarization have two independent channels, each of which operates on linearly polarized waves. A right-handed circularly polarized patch antenna with a filtering feeding network and high out-of-band radiation rejection level was proposed in [10]. A highselectivity power divider was designed using two shunted bandpass filters. Through adjusting the lengths of the two outputs of the filtering power divider, a $90^{\circ}$ phase difference was realized between the outputs. The patch and feeding network were connected by metallic pins. The measured axial ratio was below $3 \mathrm{~dB}$ in the operating band with a center frequency of $2 \mathrm{GHz}$.

The structure in [11] was a multiple-input AF in the form of a multilayer printed circuit, which was fabricated using SIW technology. It featured a dual-mode RR operating on orthogonal linear polarizations. The circular polarization was provided using a quadrature-balanced power divider (BPD) feeding two channels. A similar scheme was used in the AF proposed in [12]. It contained a dual-mode RR and two NRRs. In [12], a variant of a circular polarized AF with one NRR was described. It operated in the same manner as printed circularly polarized antennas with a single feed point [13]. The disadvantage of such antennas is the degradation of their polarization characteristics in the frequency band, which is much narrower than the gain frequency band. A scheme with two independent channels is capable of maintaining circular polarization in broader bandwidths.

This paper is a further development of the work in [12]. The device under study contains a dual-mode RR coupled with two NRRs. Two channels of orthogonal linear polarizations are connected to the outputs of a quadrature BPD. The device is intended for use in a receiver of GPS, GLONAS, and Galileo signals; its operating frequency band should not be less than $1.57-1.615 \mathrm{GHz}$, whereas the dimensions must not exceed $25 \times 25 \times 8 \mathrm{~mm}$ (hereinafter, all dimensions are given in $\mathrm{mm}$ ).

The remainder of this paper is organized as follows. Section 2 describes the AF design; Section 3 proposes an equivalent circuit for the circular polarized $\mathrm{AF}$, which describes the main tuning features of the AF that provide a frequency response close to the Chebyshev characteristic; Section 4 presents the results of the electromagnetic simulations; Section 5 compares the measured characteristics of the prototype with the results of numerical simulations; and finally, Section 6 summarizes the concluding remarks.

\section{AF Design}

Fig. 1 presents schematics of the proposed AF's design. The $\mathrm{RR}$ is made in the form of a printed half-wave patch radiator. Both NRRs are quarter-wave printed radiators. The thickness $h_{r}$ of the RR dielectric substrate should be larger than the thickness $h_{n}$ of the NRR dielectric substrate. In this case, the radiation Q-factor of the RR is much smaller than that of the NRRs. This means that the radiation pattern of the AF is practically determined using the radiation pattern of the RR. To minimize the AF's dimensions, using dielectric materials with high relative permittivity values $\varepsilon_{r}$ $>40$ is necessary.
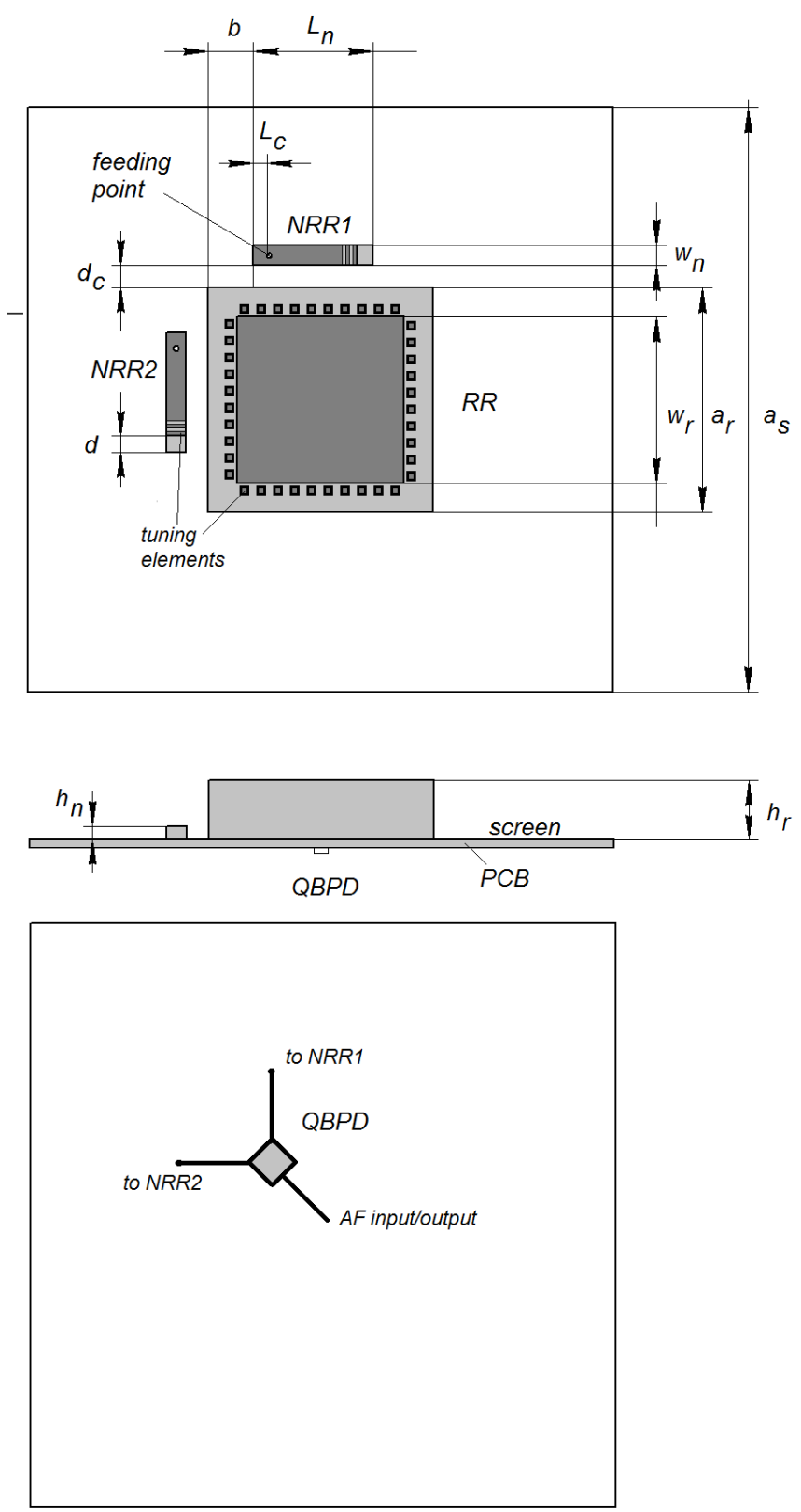

Fig. 1. Schematics of the circular polarization AF.

The resonators are placed on the upper metal surface of the grounded PCB, whereas the quadrature BPD is placed on the lower dielectric surface. The BPD outputs are connected 
to the feed points of the NRRs through holes. The NRRs are fed by small metal pins with diameters of $0.4 \mathrm{~mm}$ located at distance $L_{c}$ from the resonator edge.

The coupling between the RR and both NRRs is provided by overlapping their near fields and determined mainly by the distance $d_{c}$. The parameter $L_{c}$ determines the location of the feeding points of both NRRs, whereas $d$ is the length of the nonmetallized area on the NRRs' upper surface.

\section{AF Model in the Form of an Equivalent Circuit}

Applying the conventional approach [2] to synthesizing the considered AF would not provide satisfactory results. This is because the classical theory of filter synthesis assumes the identity of the resonators and their high Q-factors, whereas in the AF in Fig. 1, the RR and NRRs are highly different in terms of structures and electrical parameters. First, this refers to their Q-factors. The volume of the RR may exceed the volume of the NRRs by several orders of magnitude. Hence, their Q-factors $Q_{01,2}$ caused by the thermal losses are substantially different. As a rule, $Q_{02}>>Q_{01}$, where $Q_{01}$ and $Q_{02}$ are the NRR and RR thermal Q-factors, respectively (in the considered design, $Q_{01}=116$ and $Q_{02}=643$; see below). Thus, the thermal loss factor significantly affects the AF frequency response and must be considered when choosing its parameters.

Additionally, AF tuning performed using a numerical model or manually using a prototype is quite a complex and time consuming procedure. To facilitate and accelerate it, it is necessary to use a relatively simple model for determining the potentially achievable characteristics and main relationships between the figures of merit. For a simplified analysis, we propose an AF channel model in the form of the equivalent circuit in Fig. 2. The elements $L_{l}, C_{l}$, and $g_{l}$ form the first resonant contour modeling the NRRs, and the elements $L_{2}, C_{2}$, and $g_{2}$ form the second resonant contour corresponding to the RR. The capacitance $C_{c}$ is the coupling element between the two contours. The conductance $g_{r}$ emulates the energy transfer into free space through the radiation of the RR. Resistance $Z_{0}=1 / g_{0}$ is the internal resistance of the voltage source $E$.

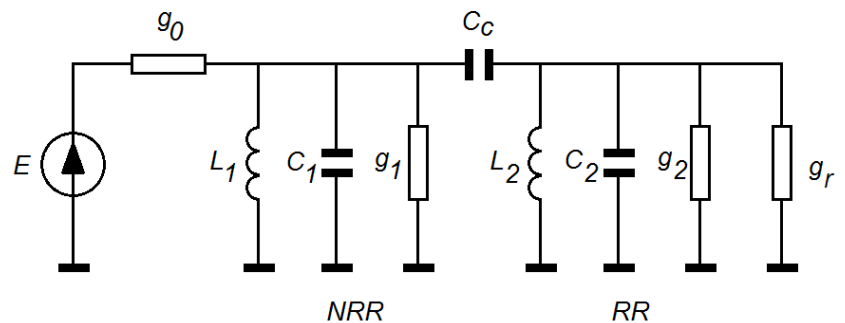

Fig. 2. AF equivalent circuit.

The primary parameters of the equivalent circuit shown in Fig. 2 are inconvenient for description because they are difficult to measure. Next, we use the generalized parameters that are traditionally used in the theory of filter synthesis based on coupled resonators [2]. These include the eigen resonant frequencies $f_{01,2}$ of the uncoupled resonators as well as the unloaded Q-factors $Q_{01,2}$, loaded Q-factors $Q_{L 1,2}$, coupling coefficient $k_{c}$, and characteristic resistances $\rho_{1,2}$. Given $g_{0}$, the parameter $\rho_{1}$ for the fixed values of the NRR Q-factors is uniquely determined using $f_{01}$ and $Q_{L 1}$. Thus, only $\rho_{2}$ is an independent variable. We define it using the ratio $k_{\rho}=\rho_{2} / \rho_{1}$. The coupling coefficient is determined through the eigen resonant frequencies of the coupled resonators $f_{r 1,2}$ [2]. Note that the generalized parameters, with the exception of the coefficient $k_{\rho}$, can be determined relatively simply from measurements or numerical modeling. As shown below, the frequency response of the synthesized AF is practically independent of $k_{\rho}$, and hence, is a function of the variables that can be determined for the real AF structure. Let $f_{0}$ be the center frequency of the AF passband of the width $\delta f, R$ be the flatness of the AF response in the passband, $L_{m}$ be the minimal insertion loss in the passband, and $K$ be the slope of the frequency response outside the passband. The parameter $K$ is defined as the ratio of the AFC values at the band edges and at the $0.1 \mathrm{GHz}$ offset.

The goal is to achieve a Chebyshev-type frequency response with a given value of the parameter $R$. For such a frequency response, the bandwidth is determined in a standard manner in terms of the level $L_{m}+R$.

However, the AF parameters are not equivalent in view of the tuning. Some can be changed quite simply in the measurements, including the eigen frequencies $f_{01,2}$, which are controlled by the tuning elements (see Fig. 1); the NRR loaded Q-factor $Q_{L 1}$, which is determined by the distance $L_{c}$; and the coupling coefficient $k_{c}$ depending on the distance $d_{c}$. We assume they are free parameters, whereas the others are fixed. By varying these generalized parameters, we can satisfy four conditions imposed on the frequency response of the equivalent circuit. The frequency response is determined as the amplitude of the transmission coefficient $T$ from the source with the internal resistance $Z_{0}$ to the load $g_{r}$.

Let us require that the function $T(f)$ satisfies the following conditions: the center frequency of the bandwidth is $f_{0}$, the frequency response has a symmetrical shape relative to the center frequency, the passband bandwidth is $\delta f$, and the value of the insertion loss $L_{m}$ should be minimal. Mathematically, the problem reduces to the solution of the system of transcendental equations. This allows us to draw some important conclusions. The symmetry requirement can be satisfied at different eigen frequencies $f_{01,2}$. The main factor determining their difference is the ratio of the characteristic resistances $k_{\rho}$. At $k_{\rho}=1$, we have $f_{01}=f_{02}$.

An optimal $Q_{L 1}$ value exists that provides the minimum value of $L_{m}$. Notably, this optimal $Q_{L 1}$ value is substantially lower than the $Q_{L 2}$ value related to $\mathrm{RR}$, unlike the classical filter theory that assumes the equality of Q-factors.

The AF parameters at the optimal $Q_{L 1}$ value versus the fractional bandwidth are shown in Figs. 3 and 4. In Fig. 3, curves $1-3$ are obtained for $f_{0}=1.6 \Gamma \Gamma ц$, and $Q_{01}=100$ (curve 1), 150 (curve 2), and 200 (curve 3); $Q_{02}=1000$; and $Q_{r}=60$, where $Q_{r}$ is the RR radiation Q-factor. The similar curves in Fig. 4 were calculated at $Q_{r}=50$ (curve 1), 60 (curve 2), 70 (curve 3), and $Q_{01}=150$. As noted above, the results shown in Figs. 3 and 4 do not depend on $k_{\rho}$, and furthermore, they do not depend on $Q_{02}$, assuming $Q_{02}>>Q_{r}$. 


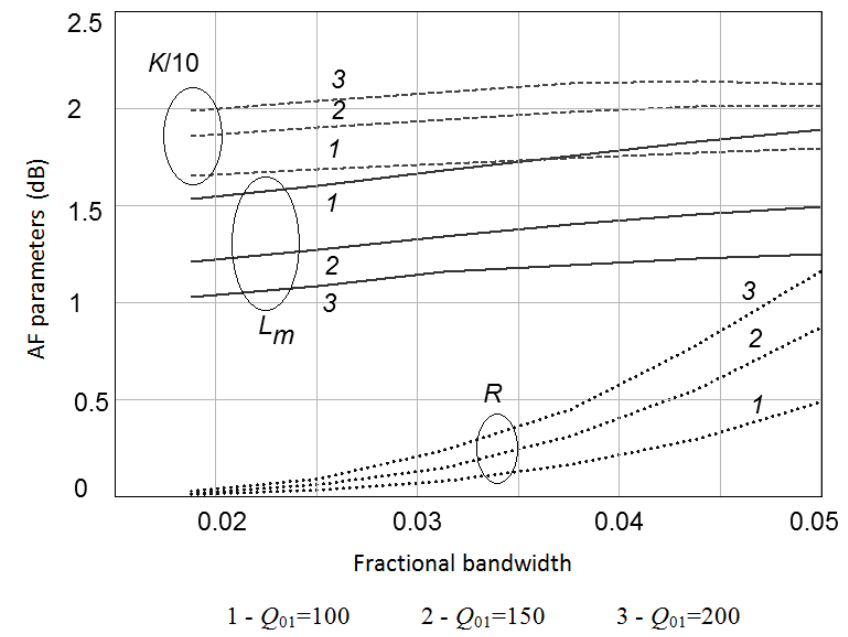

Fig. 3. AF parameters vs. fractional bandwidth at $f_{0}=$ $1.6 \mathrm{GHz}, Q_{02}=1000, Q_{r}=60, Q_{01}=100$ (curve 1), 150 (curve 2), and 200 (curve 3 ).

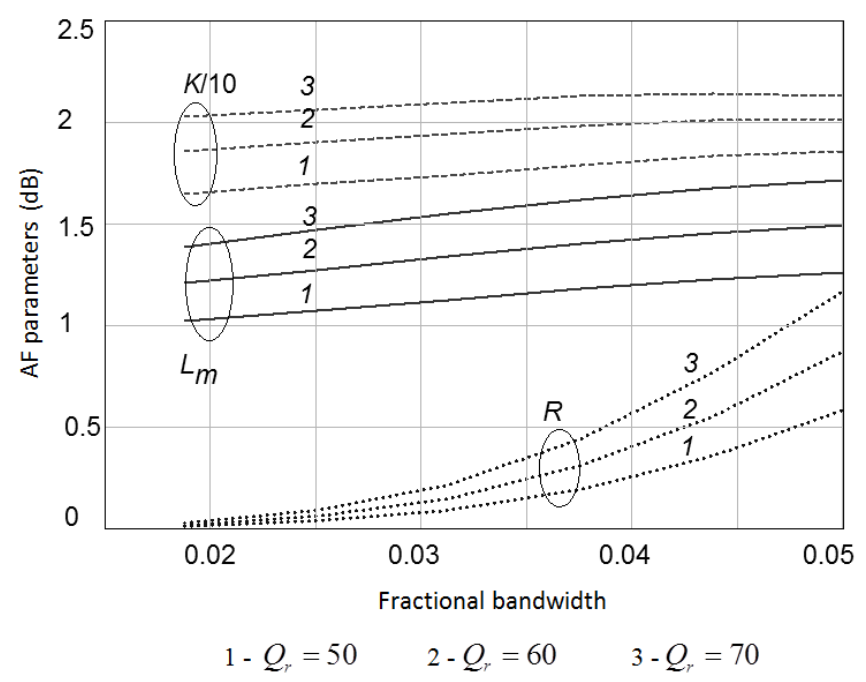

Fig. 4. AF parameters vs. fractional bandwidth at $f_{0}=1.6$ $\mathrm{GHz}, Q_{02}=1000, Q_{01}=150, Q_{r}=50$ (curve 1), 60 (curve 2), and 70 (curve 3 ).

It can be observed that some plots describing the AF properties demonstrate nonstandard behavior. In particular, the losses in the passband increase with its width, whereas they decrease in the conventional nonradiating filter. Moreover, substantial variations can be observed in the frequency response magnitude within the passband from values lower than $0.1 \mathrm{~dB}$ to those around $1 \mathrm{~dB}$. At $R<1$, the frequency response can be considered rather maximally flat compared with the Chebyshev type.

Furthermore, the losses in the passband depend on $Q_{01}$ and $Q_{r}$. They decrease as the NRR Q-factor $Q_{01}$ increases, and they increase as the radiation Q-factor $Q_{r}$ increases, which mainly determines the RR's Q value assuming $Q_{02} \gg Q_{r}$. Such behavior is attributed to the adopted tuning method that uses only the limited set of parameters. For instance, we cannot freely vary the RR loaded $Q_{L 2}$ value determined by its size. By contrast, the resonant frequency can be tuned and controlled quite simply using tuning screws.

The curves in Figs. 3 and 4 provide a preliminary assessment of the AF figures of merit achieved at typical parameters of the resonators. For instance, with the passband bandwidth $\delta f=60 \mathrm{MHz}$, it is possible to achieve slopes of $K \approx 18 \mathrm{~dB}$ at a loss value of $L_{m}=1.2 \mathrm{~dB}$ and flatness of $R=0.2 \mathrm{~dB}$.

\section{Electromagnetic Modeling}

To reduce the AF's size, we used high-Q ceramic material with a dielectric permittivity of $\varepsilon_{r}=45$ and $\operatorname{tg} \delta=10^{-3}$. Some of the resonator sizes were fixed: $h_{r}=8, h_{n}=2, w_{n}=2, a_{r}=$ 19. The goal of EM modeling was to determine the AF parameters that could provide the following characteristics: $\delta f>60 \mathrm{MHz}, f_{0}=1.6 \mathrm{GHz}$, minimum loss in the bandwidth, and minimum radiation of the cross left-handed circular polarization (LHCP), provided that the right-handed circular polarization (RHCP) was dominant.

At first, the characteristics of the uncoupled NRRs and RR were investigated. The aim was to tune them to the center operating frequency and determine the thermal and radiation Q-values. For the numerical tuning, the parameters $L_{n}, d$, and $w_{r}$ were varied (see Fig. 1). Computations were performed using the eigen modes engine in HFSS simulator software (ANSYS, Inc., Canonsburg, PA, USA). In this regime, the eigen complex frequencies were computed for the resonators surrounded by an air box bound by perfectly matched layers. The imaginary part of the eigen frequency determines the Q-factor of the resonator.

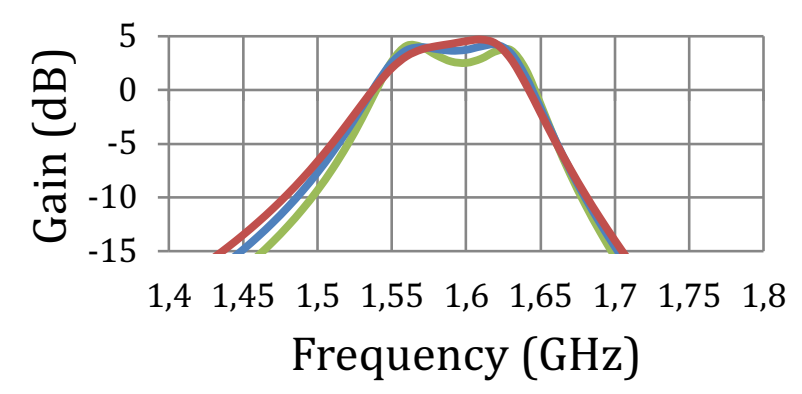

$\mathrm{Lc}=0,9 \mathrm{~mm} \longrightarrow \mathrm{Lc}=1,1 \mathrm{~mm} \longrightarrow \mathrm{Lc}=1,3 \mathrm{~mm}$

a

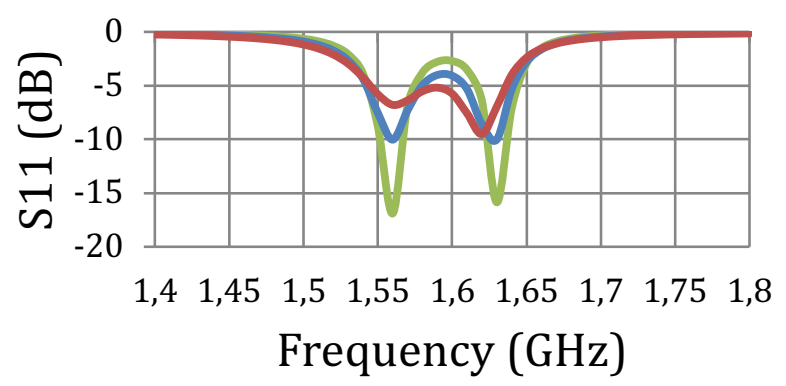

$\longrightarrow \mathrm{Lc}=0,9 \mathrm{~mm} \longrightarrow \mathrm{Lc}=1,1 \mathrm{~mm} \longrightarrow \mathrm{Lc}=1,3 \mathrm{~mm}$ 


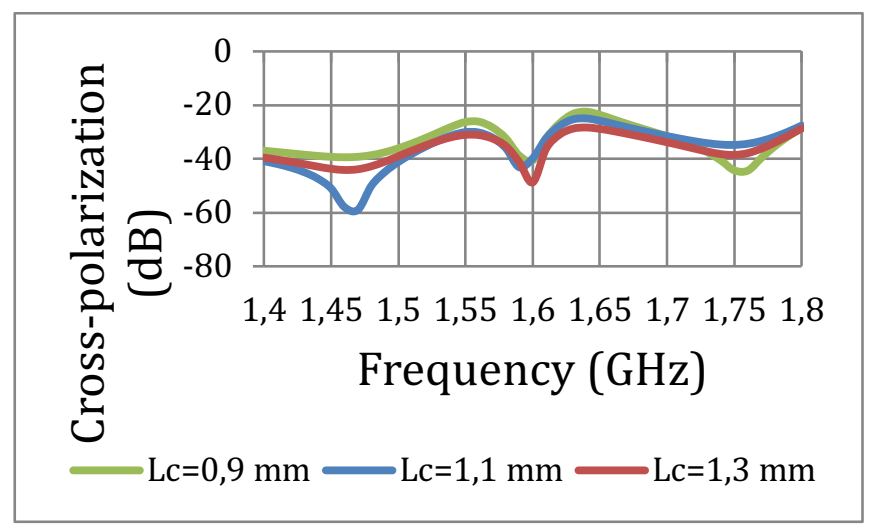

c

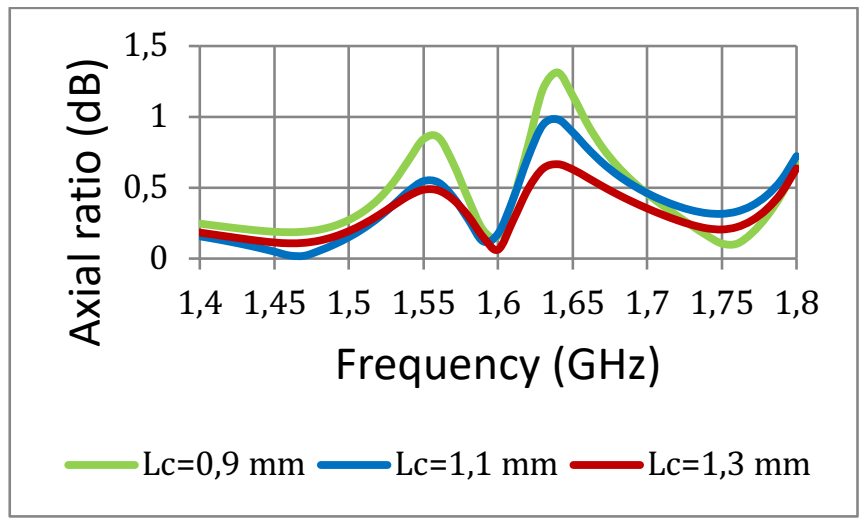

d

Fig. 5. (a) gain, (b) reflection coefficient, (c) crosspolarization, (d) axial ratio vs. frequency at $L_{c}=0.9,1.1$, and $1.3\left(d_{c}=3.5\right)$

In general, an unloaded $\mathrm{Q}$ value is determined by two factors: $Q^{-1}=Q_{r}{ }^{-1}+Q_{h}{ }^{-1}$, where $Q_{r}$ and $Q_{h}$ correspond to the radiation and heat loss, respectively. The simulations showed that at a resonant frequency around $1.6 \mathrm{GHz}$, the total unloaded NRR Q-factor was $Q_{1}=112$, which was determined using the radiation and thermal factors $Q_{r l}=$ 2970 and $Q_{01}=116$, respectively. For the RR, $Q_{2}=91, Q_{r 2}=$ 106 , and $Q_{02}=643$.

Calculations using the method described in Section 3 showed that having such Q-factors and $\delta f=60-80 \mathrm{MHz}$, the minimum loss value in the passband was $L_{m}=2.25-2.4 \mathrm{~dB}$.

Next, the external parameters of the AF were computed: the gain $G$, reflection coefficient $S_{11}$, cross-polarization $C$, and axial ratio $A R$. The driven mode regime of the AF was modeled using two lumped ports placed in small gaps between the pins and ground plane.

The polarization characteristics were controlled in the direction of maximum radiation along the $0 Z$ axis. The RHCP radiation was provided by the simultaneous excitation of both NRRs with a phase shift of $90^{\circ}$ between the two.

The parameter $C$ was defined as $C=\sqrt{P_{L H C P} / P_{R H C P}}$, where $P_{L H C P}$ and $P_{R H C P}$ were the radiation powers at the cross- and co-polarizations in the $z$-direction. Fig. 5(a-d) show the frequency dependencies of the characteristics $G$, $S_{11}, C$, and $A R$ at different positions $L_{c}$ of the excitation points (see Fig. 1).

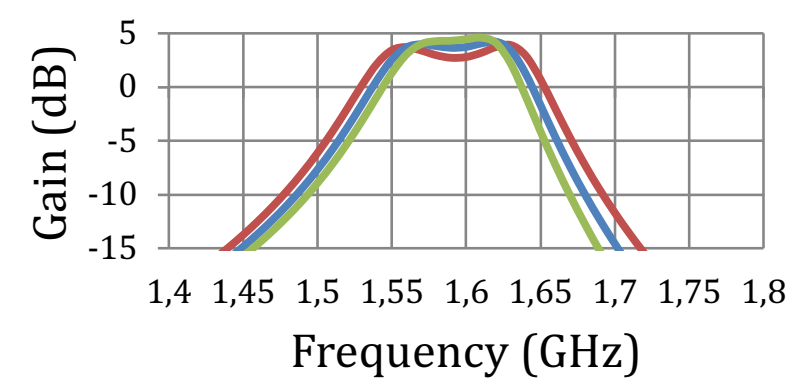

$\longrightarrow \mathrm{dc}=2,5 \mathrm{~mm} \longrightarrow \mathrm{dc}=3,5 \mathrm{~mm} \longrightarrow \mathrm{dc}=4,5 \mathrm{~mm}$

a

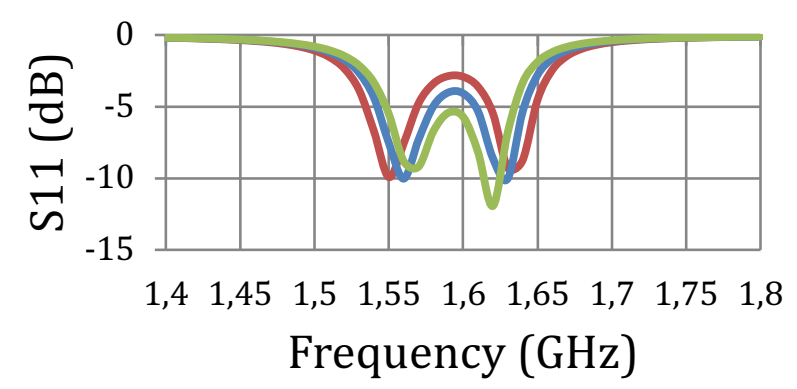

$\longrightarrow \mathrm{dc}=2,5 \mathrm{~mm} \longrightarrow \mathrm{dc}=3,5 \mathrm{~mm} \longrightarrow \mathrm{dc}=4,5 \mathrm{~mm}$

b

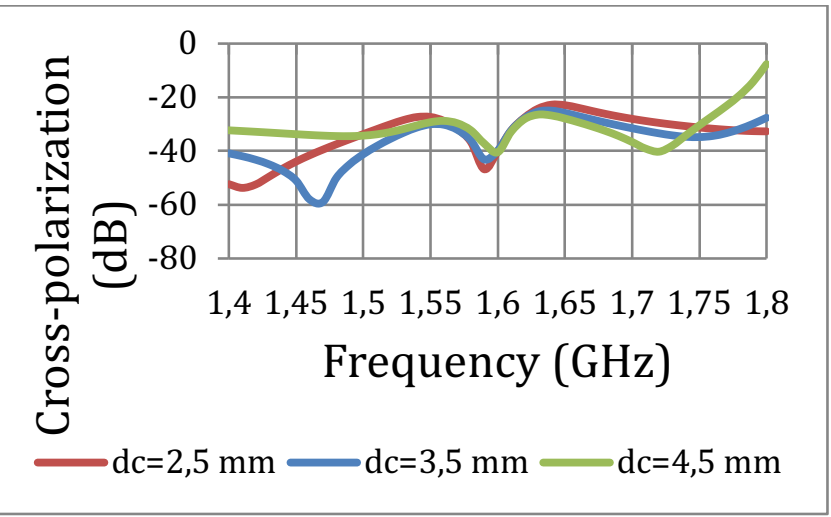

c

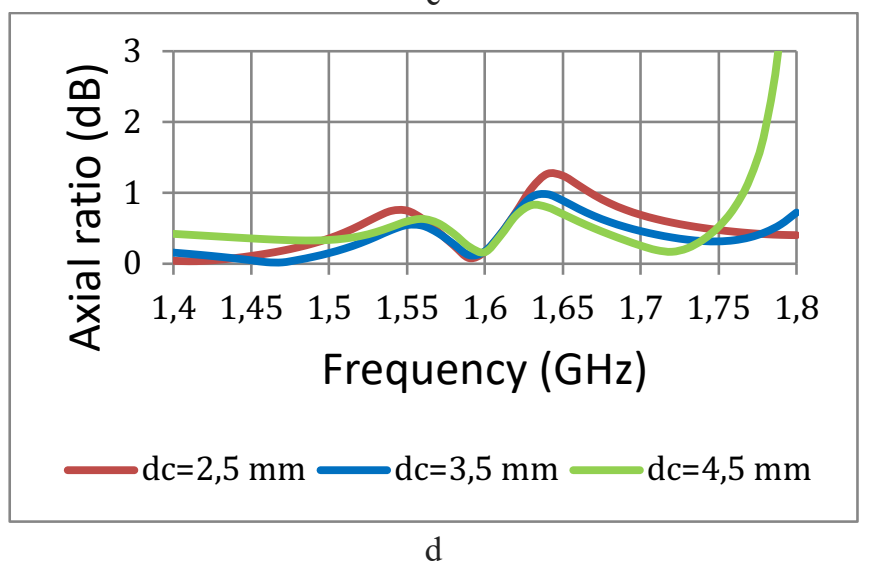

Fig. 6. (a) Gain, (b) reflection coefficient, (c) crosspolarization, and (d) axial ratio vs. frequency at $d_{c}=2.5,3.5$, and $4.5\left(L_{c}=1.1\right)$. 
The curves in Fig. 5(a-d) were computed for the three positions $L_{c}=0.9,1.1$, and 1.3; other parameters were $d_{c}=$ 3.5, $L_{n}=11, a_{r}=19, a_{s}=100, w_{r}=11.2, w_{n}=2, h_{r}=8, h_{n}=$ $2, d=1.3, b=4, \varepsilon_{r}=45$, and $\operatorname{tg} \delta=0.001$. Fig. 6 (a-d) show the same characteristics versus frequency computed for the three values of $d_{c}=2.5,3.5$, and 4.5 at $L_{c}=1.1$; the other parameters were the same.

The frequency dependencies in Figs. 5 and 6 have the expected character. The change in position of the feed point mainly changed the loaded NRR $Q_{L 1}$ value, which in turn led to the change in the Q-factors for the eigen modes of the NRR-RR coupled system. Decreasing the Q-factors decreased the ripples in the passband as well as decreased the cutoff rate outside it. Notice the asymmetry of the frequency response arising at the larger $L_{c}$. This is because of the influence of the feed point position on the NRR resonant frequency. To maintain the symmetry, the resonant frequency should be tuned.

Fig. 6(a-d) shows that the distance $d_{c}$ mainly affected the coupling between the resonators; that is, the difference between the resonant frequencies of the NRR-RR eigen modes. This parameter determines the bandwidth of the passband. The bandwidth widening that occurred at smaller $d_{c}$ led to larger ripples in the passband. Note that all the trends in dependencies of the AF characteristics found in the numerical simulations are qualitatively the same as those obtained in the simplified equivalent circuit model.

In addition, note that the level of the reflection coefficient at the center frequency is quite high. This is because of the relatively high radiation $\mathrm{Q}$ value of the patch antenna $Q_{r 2}=106$ (see above). Having such a $Q_{r}$ value, the required bandwidth can only be obtained by some mismatch at the center frequency, resulting in an increase in insertion loss.

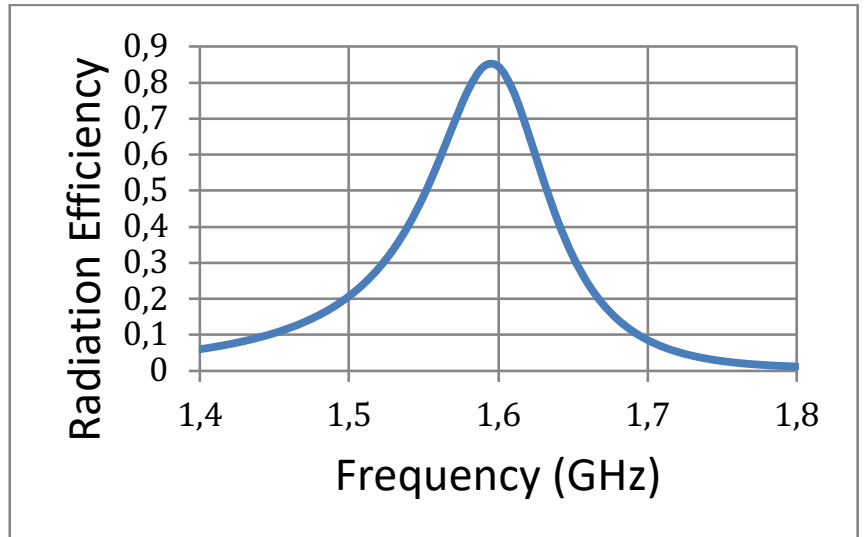

Fig. 7. Radiation efficiency vs. frequency $d_{c}=3.5, L_{c}=1.1$

The polarization characteristics only depend weakly on both $d_{c}$ and $L_{c}$. They are mainly determined by the distance $b$ (see Fig. 1). The level of cross-polarization in the passband does not exceed $-25 \mathrm{~dB}$. Fig. 7 plots the simulated radiation efficiency in the examined frequency range, where the maximum resonant value is approximately $85 \%$.

The computed surface current magnitude distributions on the metal screen are shown in Fig. $8\left(\mathrm{a}^{-} \mathrm{c}\right)$ at three different frequencies; 1.4 and $1.8 \mathrm{GHz}$ lie outside the passband, whereas $1.6 \mathrm{GHz}$ lies in the middle of the passband. It can be observed that in the stopband regime, the current magnitude in the area under the RR is relatively small, and in the passband, it is approximately two orders of magnitude greater.
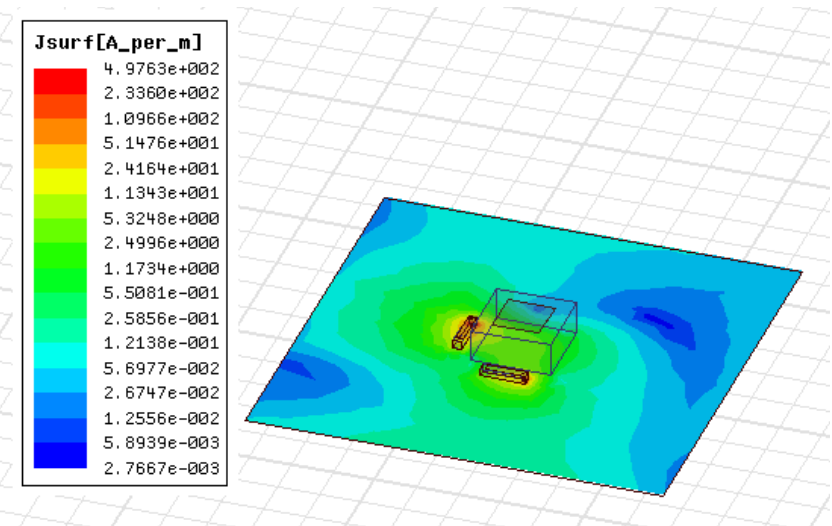

a

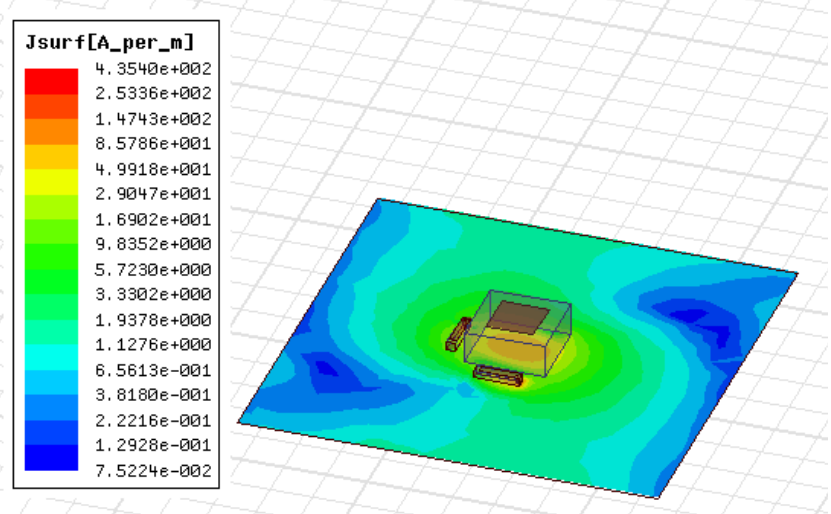

b
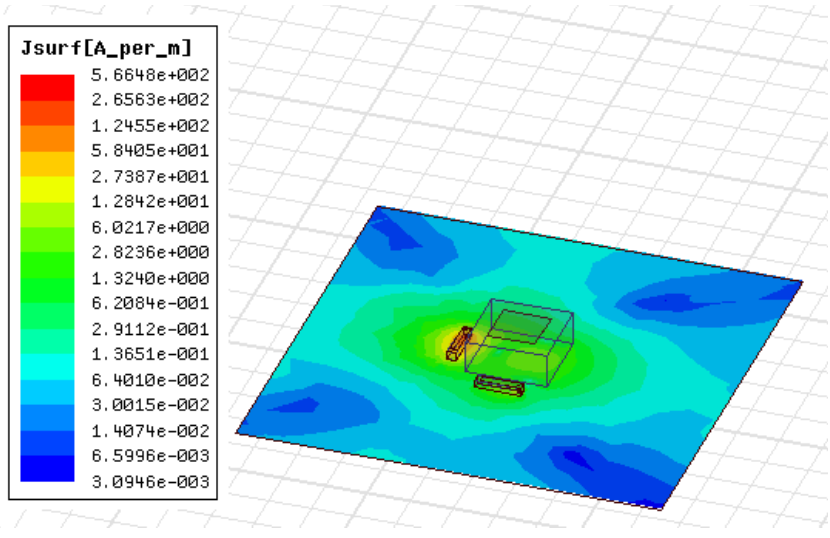

c

Fig. 8. Surface current distribution on the metal screen at $f=$ 1.4 GHz (a), 1.6 GHz (b), and 1.8 GHz (c).

Such an increase means that in the passband, the NRRs and RR are strongly coupled and the AF effectively radiates energy into free space. Outside the passband, the coupling between the NRRs and RR is low and the AF does not radiate energy. 


\section{Experimental Study}

The AF sample was made and tested, as shown in Fig. 9, with the following parameters: $L_{c}=1.1, L_{n}=11, a_{r}=19, a_{s}=$ $100, w_{r}=11.2, w_{n}=2, h_{r}=8, h_{n}=2, d=1.3, b=4, d_{c}=3.5$, $\varepsilon_{r}=45$, and $\operatorname{tg} \delta=0.001$. The sample was fabricated on a fiberglass printed circuit board. On its front side, the RR and NRRs were arranged, whereas the quadrature BPD was placed on the backside. It was designed using the QCN-19 chip.

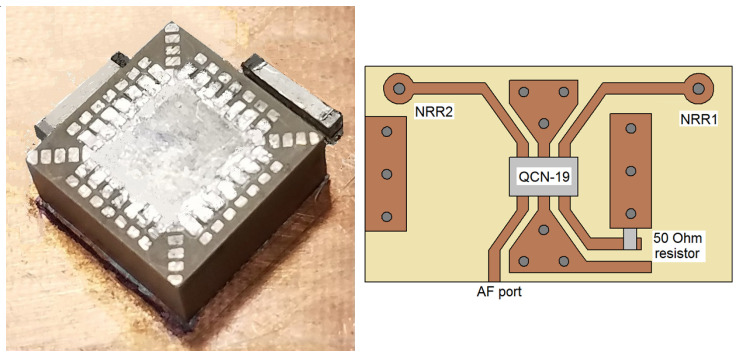

Fig. 9. AF sample: left - front side photo, right - QBPD circuit topology.

The frequency characteristics of the gain $G$ and crosspolarization $C$ were measured. The antenna input reflection coefficient was also measured but is not presented here. Because the quadrature BPD absorbed the waves reflected from the NRR, the amplitude of the reflected wave measured in the antenna port of the QBPD was negligible. Therefore, the reflections from NRRs 1 and 2 could not be measured and compared with the simulated ones in this manner. In addition, the radiation pattern was measured at the center frequency. The measurements were performed in an anechoic chamber.

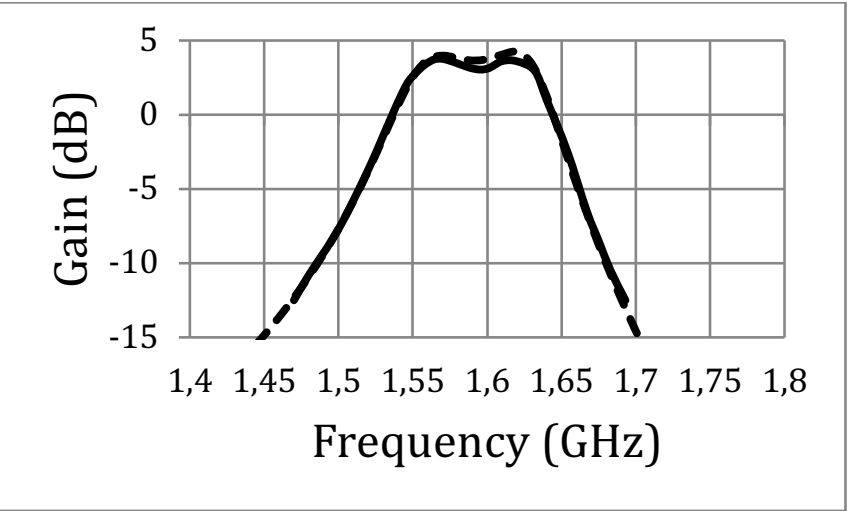

Fig. 10. Gain vs. frequency in the vicinity of the passband: solid - measured, dashed - simulated.

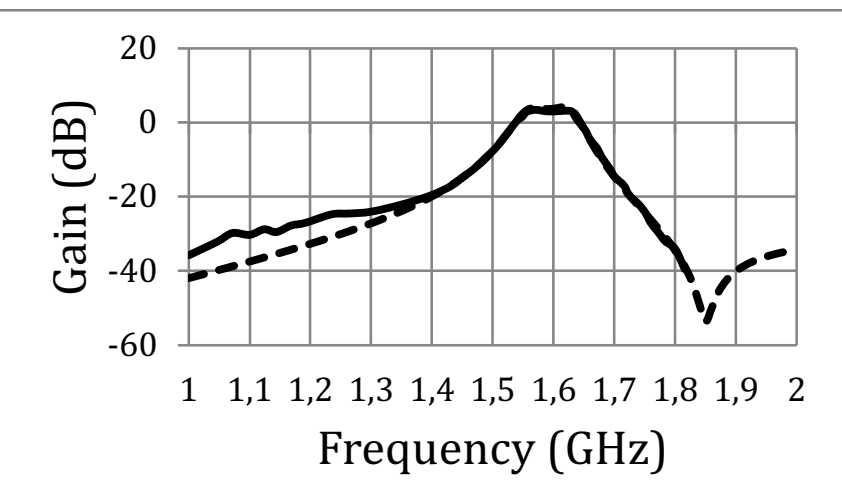

Fig. 11. Gain vs. frequency in the extended frequency band: solid - measured, dashed - simulated.

A two-polar wideband Lindgreen antenna in the transmission regime and an Agilent VNA N5230C were used, allowing simultaneous measurements of the antenna parameters for two orthogonal circular polarizations. To measure the AF gain, a standard technique was employed that was based on replacing the antenna being tested with a reference antenna of known gain. In this case, a half-wave vibrator was used.

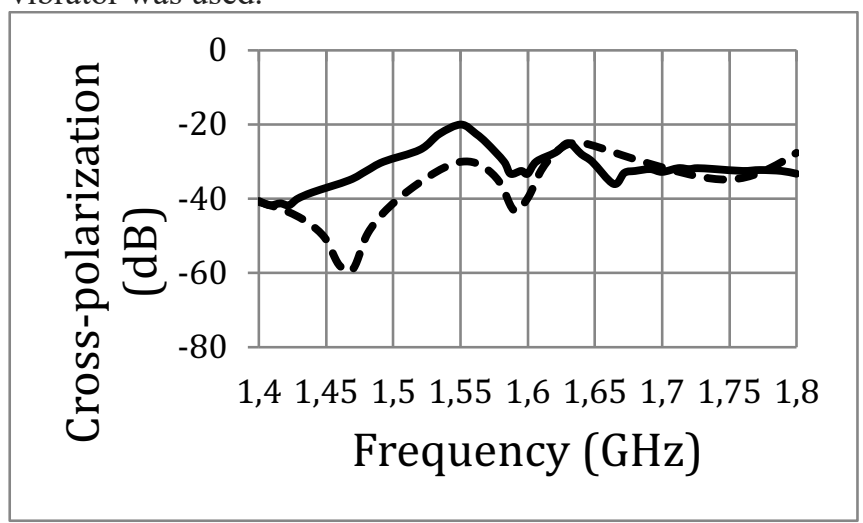

Fig. 12. Polarization ratio vs. frequency: solid - measured, dashed - simulated.

Figs. 10-12 present the results of the measurements (solid lines) compared with the EM simulation results (dashed lines). The frequency response of the gain in the vicinity of the AF passband is shown in Fig. 10. The same frequency response in a wider frequency band is presented in Fig. 11, showing the out-of-band attenuation. A good agreement between the simulated and measured results can be observed.

Fig. 13 presents the normalized radiation pattern in the RHCP radiation mode at $1.6 \mathrm{GHz}$. The radiation pattern is shown as a function of the elevation angle $\theta$ in the ZOX cut plane corresponding to the azimuth angle $\varphi=0$. Notably, 


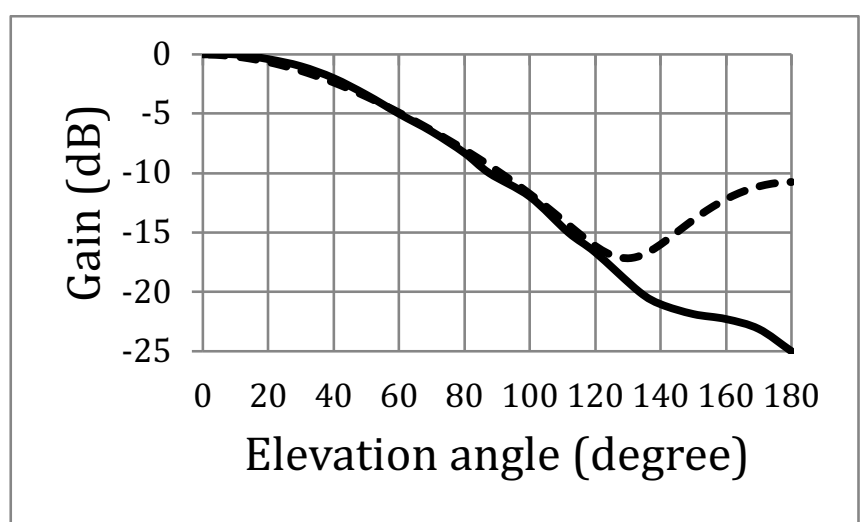

Fig. 13. RHCP normalized radiation pattern at $1.6 \mathrm{GHz}$ : solid - measured, dashed - simulated.

in this case, the radiation pattern was practically independent of the angle $\varphi$. With the measured and computed patterns, it was possible to determine the maximum directivity value of $6.6 \mathrm{dBi}$ at $\theta=0^{\circ}$. The measured gain value was $4.1 \mathrm{dBi}$, resulting in a total insertion loss of $2.5 \mathrm{~dB}$. From the other side, the estimated insertion loss obtained in Section 3 was 2.25-2.4 dB. Both loss values are close. This can serve as an additional confirmation that the equivalent circuit model is not only qualitatively correct but can also provide the correct quantitative description.

\section{Conclusions}

This study demonstrated that the proposed AF design can be used as a filtering antenna with the frequency response of a second-order bandpass filter for a multisystem receiver of GPS, GLONAS, and Galileo signals. The insertion loss level does not exceed similar values in any module of the traditional configuration, in which a second-order bandpass filter is followed by a printed antenna. This study's results can be considered as proof of the proposed design concept for a compact AF of circular polarization with an increased frequency band and low level of cross-polarization in the passband.

\section{Acknowledgements}

The authors would like to thank the staff of the Kompas Bureau, who helped in the manufacturing of the prototype as well as the measurements.

\section{References}

[1] L. J. Chu, Physical limitations of omnidirectional antennas, Journal of Applied Physics 19 (1948), pp.1163-1175.

[2] G. Mattaei, L. Young and E.M.T. Jones, Microwave Filters, Impedance-Matching Networks, and Coupling Structures, Artech House, Narwood, 1980.

[3] J. Zuo, X. Chen, G. Han, L. Li, and W. Zhang, An Integrated Approach to RF Antenna-Filter Co-Design, IEEE Antennas Wireless Propag Lett 8 (2009), pp.141144.
[4] J. Jadhav, P. Deore, Filtering antenna with radiation and filtering functions for wireless applications, Journal of Electrical Systems and Information Technology 4 (2017), pp.125-134.

[5] S. Chen, Y. Zhao, M. Peng and Y. Wang, A Codesigned compact dual-band filtering antenna with PIN loaded for WLAN applications, International Journal of Antennas and Propag., (2014), Article ID $826171 \mathrm{http} / / /$ doi.org/10.1155/2014/826171.

[6] M. Sabran, S. Rahim, C. Leow, P. Soh, B. Chew, and G. Vandenbosch, Compact circularly polarized truncated square ring slot antenna with suppressed higher resonances, PLOS ONE 2 (2017) https://doi.org/10.1371/journal.pone.0172162.

[7] M.K. Alkhafaji, R.K.Z. Sahbudin, A.B. Ismail and S.J. Hashim, Design of a selective filter-antenna with low insertion loss and high suppression stopband for WiMAX applications, International Journal of Sensor Networks and Data Communications, 2016, DOI: 10.4172/2090-4886.1000147.

[8] W. J. Wu, Y. Z. Yin, J. J. Xie, X. S. Ren, S. L. Zuo, Low-cost microstrip antenna with a monopole-like radiation pattern for $\mathrm{RF}$ front end, Microwave Opt. Technol. Lett. 54 (2012), pp.1810-1814.

[9] W. J. Wu, Y. Z. Yin, S. L. Zuo, Z.Y. Zhang, and J. J. Xie, A new compact filter-antenna for modern wireless communication systems, IEEE Antennas Wireless Propag. Lett. 10 (2011), pp.1131-1134.

[10] M. Qu, M. Li, L. Yao, M. Rao, S. Li, L. Deng, A novel circularly polarized filtering antenna with high out-ofband radiation rejection level, Microwave Opt. Technol. Lett. 60 (2018), pp.134-140.

[11] US patent 8,836,596 B2, Sep. 16, 2014.

[12] RF patent 2448396, Apr. 20, 2012.

[13] K. L. Wong, Compact and Broadband Microstrip Antennas, John Wiley \& Sons Inc., New York, 2002. 